\title{
Predictive Factors Affecting Newborn Survival Admitted in Special Newborn Care Units of Tertiary Care Hospitals
}

\author{
Shah Harsh ${ }^{1}$, Nagar Shashwat ${ }^{2, *}$, Thomas Ebbie ${ }^{3}$, Patel Jay ${ }^{4}$
}

\begin{abstract}
Context: Government efforts are being made for the reduction of child mortality through the facility and community-based strategies. Studies have indicated that several other factors also have an impact on child survival. This calls for researches to be directed efforts and understanding of the local causes and factors associated with it. Aim: To analyze the sociodemographic, programmatic and clinical profile of neonates' admitted in SNCUs. Settings and Design: A cross sectional study amon 43 SNCUs during year 2017-18. Methods and Materials: The data was recorded from the pre-defined case record registers and analyzed using simple proportions. Statistical Analysis used: Chi-square test and multivariable logistic regression analysis were carried out for finding the association between survival rate of neonates and other variables. Results: Out of 48,775 neonates admitted at selected SNCUs across the State, $68.5 \%$ were successfully discharged, $6.7 \%$ referred to higher centers, $10 \%$ left against medical advice and $15.2 \%$ died during the study period. Survival Rate was observed to be significantly associated with the Place of Delivery, Mode of Transport, Type of Admission, Maturity of child admitted, weight of a child during admission and Indications of admission. Key words: SNCU, Child Survival, Regression, NMR, U5MR, Gujarat state.

Key Messages: Several factors influence the new-born survival which must be given attention along with the programme interventions. These factors must be studied more indepth and may be given due weightage by the programme managers to enhance new-born survival.
\end{abstract}

Shah Harsh ${ }^{1}$, Nagar

Shashwat ${ }^{2, *}$, Thomas

Ebbie $^{3}$, Patel Jay ${ }^{4}$

'Senior Consultant, NHM, Government of Gujarat, Gujarat, INDIA.

${ }^{2}$ Department of Community Medicine, PIMSR, Parul University, Vadodara,

Gujarat, INDIA.

${ }^{3}$ Statistician Cum Tutor, NAMO MERI,

Silvassa, INDIA.

${ }^{4}$ Consultant, Nutrition Consultant,

NHM, Govt. of Gujarat, Gujarat, INDIA.

\section{Correspondence}

Dr. Shashwat Nagar

Associate Professor, Community Medicine, Parul Institute of Medical Sciences and Research, Medical Teaching Staff quarters, Parul University, Vadodara-391760, Gujarat, INDIA.

Mobile no: +91-9998606749

Email: ssnagar.md@gmail.com

History

- Submission Date: 30-06-2020

- Revised Date: 20-07-2020

- Accepted Date: 18-09-2020

DOI : 10.5530/ijmedph.2020.4.40

Article Available online

http://www.ijmedph.org/v10/i4

\section{Copyright}

(c) 2020 Phcog.Net. This is an openaccess article distributed under the terms of the Creative Commons Attribution 4.0 International license.

\section{INTRODUCTION}

Millions of children die every year because of preventable or treatable causes despite the progress over the last two decades. While concentrated efforts aimed at improving child survival received large reductions in mortality levels among children under 5 years of age in recent years, still, priorities are to be set to address the specific survival needs of children to reduce further deaths. Sustainable Development Goal (SDG) 3, target 3.2 suggests for an end to preventable deaths of new-borns and children under 5 years of age and specifies that all countries should aim to reduce neonatal mortality to at least as low as 12 deaths per 1,000 live births and under-five mortality to at least as low as 25 deaths per 1,000 live births by $2030 .{ }^{1}$ During 2000-2017, neonatal mortality (NMR; deaths within the first 28 days of life) declined by 41 per cent and in 2017 alone, 5.4 million children died before reaching their fifth birthday. Globally, an estimated 2.5 million new-borns died in the first month of life in 2017 - approximately 7,000 every day - most of whom died in the first week after birth. ${ }^{2}$

According to various rounds of the National Family Health Survey (NFHS) data, the under-five mortality rate (U5MR) has declined by a little more than half in the past 23 years, namely, from 109 deaths (per 1000 live births) in c.1990 (five years prior to

the 1992-1993 NFHS) to 50 deaths (per 1000 live births) in c.2013 (five years prior to the 2015-2016 NFHS). ${ }^{3}$ For the same period, the reduction in the neonatal mortality rate (NMR) was only 19 units (per 1000 live births). ${ }^{3,4}$ Thus, if India makes progress towards a similar decline in the next 12 years, it is very likely to meet the target set by the Sustainable Development Goals (SDGs) for U5MR but unlikely to meet the NMR target. Neonatal mortality rate of Gujarat accounts for $63 \%$ of total under five aged children mortality. Gujarat has achieved U5MR of 33 per 1000 live births, IMR of 30 per 1000 live births and 21 per 1000 live births NMR. ${ }^{5}$ Gujarat has achieved considerable progress in maternal and child survival rates with available resources. There are very few studies that provide comprehensive overview of neonatal morbidity and mortality data with factor associated with them.

For a country, to achieve ambitious child survival goal, requires a basic understanding of the local causes for their deaths and factors associated with it. Simple interventions aimed at these main causes have been tested and found to be effective in reducing the neonatal mortality. ${ }^{6,7}$ The existing evidenced based practices can help to reduce more than $70 \%$ of neonatal mortality, but is often challenged by an

Cite this article: Harsh S, Shashwat N, Ebbie T, Jay P. Predictive Factors Affecting Newborn Survival Admitted in Special Newborn Care Units of Tertiary Care Hospitals. Int J Med Public Health. 2020;10(4):184-8. 
uneven distribution of the service coverage in the various geographical areas. ${ }^{8}$ The facility based new-born care is crucial component but there are various programmatic and clinical components which affects the survival of the neonates.

The present study has been carried to analyse the various factors which have an impact on the neonatal survival among the SNCU admitted neonates. The various factors of the admitted neonates have been tested for to analyse their impact over the outcome in terms of neonatal survival. The aim of the study was to develop a regression model which can depict the impact of various contributing factors towards the survival of the admitted neonates. The study was conducted with the objectives to explicate the relationship of the various factors contributing to neonatal mortality and to find out the impact of various factors contributing individually and coherently towards the outcome among SNCU admitted children.

\section{SUBJECTS AND METHODS}

A cross sectional study was carried out amongst the 40 SNCUs which includes all Govt. supported Medical college attached hospitals, district hospitals and trust hospitals. These SNCUs are equipped with 15 beds to 100 beds of radiant warmer with all support system as per guidelines of SNCUs. In Gujarat, these SNCUs are supported by State Govt budget and National Health Mission. Study Population: The study subjects were 48,775 Neonates admitted in SNCUs who were delivered in same facility of SNCUs considered as "Inborn" and considered as "Outborn" if born outside. Study Period: The aggregated compiled secondary data was taken from April 2017 to March 2018. Data Collection: The monthly SNCUs reports were the source of data and due efforts were made to conceal identity of hospitals and patients. The primary data has been recorded in pre-defined case record registers of demographic, programmatic information of patients, clinical parameters of neonates and mothers filled up by paediatricians and staff nurses. It also includes information on mode of transport use during the process, place of delivery, duration of stay along with treatment outcome as discharged, expired, LAMA and referred. Inclusion Criteria: All babies who had less than 28 days of life admitted in SNCUs only with indoor registration number. Exclusion Criteria: The babies who had life of more than 28 days and not registered as indoor case. Ethical permission was sought from the concerned authority of State Health and Family Welfare, Govt of Gujarat. Limitation of analysis: The study was done on aggregated data of neonates based on Govt supported or Govt Affiliated SNCU admissions. The private owned SNCUs were not included into study. The follow up on discharged, LAMA and referred neonates were not done during the study. Data analysis: Statistical analysis was done analysing the percentages, proportions, Chi-square and multivariable logistic regression analysis using Statistical Package for the Social Sciences (SPSS) version 17 (SPSS-Inc., Chicago, US).

\section{Multi-variable Logistic Regression Analysis}

There was total 48,775 neonates admitted in SNCUs, out of which the outcome of the children who were referred or those who left against medical advice (LAMA) were removed and the multivariable logistic regression was run on the total of 40,806 cases of successfully discharged $(33,412)$ and expired $(7,394)$. A multivariable logistic regression analysis was carried out where the outcome was measured in terms of survived (successfully discharged) and non-survived (expired) among the all the children admitted in the SNCU for the said duration.

Predictors of the survival among children taken as independent variables were several variables like indication of admission, place of delivery, birth weight, maturity of the pregnancy, type of admission and mode of transport to the SNCU. Some of these variables had to be recoded for developing a significant regression model. 'Place of delivery' (PoD) variable was categorized as FRUs, PHCs, Home Delivery, Private Institutes and Others. Here FRU was taken as comparative standard. 'Mode of Transport' (MoT) variable was categorized into self-arrange (private) and Govt. provided. Here Govt. provided was taken as comparative standard. 'Type of admission' (ToA) was categorized into inborn, outborn (health facility referred) and out-born (community referred by health staff). Here inborn was taken as comparative standard. 'Maturity' was categorized into pre-term, full-term and post-term. Here pre-term was taken as comparative standard. 'Weight' was categorized into normal, low, very low and extremely low. Here Normal was taken as comparative standard. 'Indication of admission' (IoA) was categorized into any other, congenital abnormalities, hypo/hyper-thermia, hypoglycaemia, low birth weight, meconium aspiration, jaundice, prematurity, respiratory distress and birth asphyxia. Here any other was taken as comparative standard.

\section{RESULTS}

Neonatal characteristics of SNCU Admission: During the study, 48,775 neonates were admitted at selected SNCUs across the State. Out of which, 27,542 (56.5\%) neonates were inborn admission and 21,233 (43.5\%) were outborn admission. There were admission of 18 (0.03\%) ambiguous, 21347 (43.77\%) female and 27410 (56.20\%) male neonates admitted in SNCUs.

Outcome of the admitted neonates: The study found the outcome was categorized in 4 sections as successfully discharged, referred to higher centre, leave against medical advice (LAMA) and died. The analysis showed that 33,412 neonates were successfully discharged (68.5\%), 3,287 were referred to higher centres $(6.7 \%), 4,682$ were left against medical advice (LAMA) (10\%) and 7,394 were died during the study period $(15.2 \%)$. The analysis found that every $2^{\text {nd }}$ neonates was about to discharge successfully during the course of management. The study had not explored the reasons behind the LAMA and referred to higher centres as a limitation.

\section{Multi-variable Logistic Regression Analysis (Above} tables)

Dependent Variable: Outcome $=$ Not Survived $($ coded as 0$)$ and Survived (coded as 1). Hence, the outcome of "Not Survived" was taken as baseline or reference. Independent Variables were taken as below

1. Place of Delivery (PoD) (5)

2. $\quad$ Mode of Transport (MoT) (2)

3. Type of Admission (ToA) (3)

4. Maturity (3)

5. Weight (4)

6. Indication of Admission (IoA) (Categories: 10)

The chi-square test suggests that the independent variables added in this regression model was statistically significant related to dependent variable as $P<0.001$. The association was found to be statistically significant which indicates that each of these had a relationship with the outcome in terms of survival. Cox and Snell R Square model summary suggests that $18.3 \%$ of the Outcome was explained by these independent variables in this model. Out of 27 categories of all 6 variables, 6 categories were not significant and rest 21 categories were statistically significant in the model. The regression model has also shown the relationship of each of these having a direct impact on neonatal survival with beta exponential values.

Table 1 and 2 shows the male neonates formed the relative majority (56.3\%) among all the genders. The regression equation model showed 
that survival rate was higher in neonates referred from government institution was $84.8 \%$ followed by private institution $(72.6 \%)$, during commute $(70.9 \%)$ and home delivery $(66.5 \%)$. This data revealed the importance timely stabilization and care at the place of delivery. In the equation, the majority of neonates was transferred through Government provided vehicle $(53.5 \%)$ with a survival rate of $82.8 \%$. The survival rate for the inborn was $87.1 \%$ followed by the referral from the community by front line health workers $(84.5 \%)$ and referral from the other health facility $(71.6 \%)$. The survival for the neonates referred from other health facility was low as compared to other referrals which may give hypothesis for the correlation between survival of neonates and delays in different mode of transport including supportive referral system.

The survival rate in the full term showed statistically significant $(88.1 \%)$ as compared to pre-term (70.9\%) and post-term (79.4\%). The survival rate was significantly high in the normal birth weight of the neonates $(89.2 \%)$ followed by low birth weight $(86.1 \%)$, very low birth weight
(57.1\%) and extremely low birth weight (12.8\%) which was consistent with clinical scenario of new-born survival. The survival rate in neonates admitted at SNCUs with indication from jaundice was high (98.2\%) followed by hypo/hyperthermia, hypoglycaemia, meconium aspiration, congenital abnormalities and others. The survival rate is lowest in neonates admitted with indication of prematurity (37.9\%).

\section{DISCUSSION}

A number of studies have attempted to identify the contextual factors demographic, geographical factors and indirect causes associated with the childhood illnesses. ${ }^{9}$ The majority of high-risk districts for NMR and U5MR belong to the states of Assam, Bihar, Chhattisgarh, Jharkhand, Madhya Pradesh, Orissa, Rajasthan and Uttar Pradesh. The high-risk districts in NMR terms are also across rich and advanced states like Andhra Pradesh, Gujarat and Telangana. ${ }^{10}$ Socio-economic factors such as urbanization, education status and family income; coupled with early

Table 1: Categorical Variable of Neonates admitted in SNCUs $(N=40,804)$.

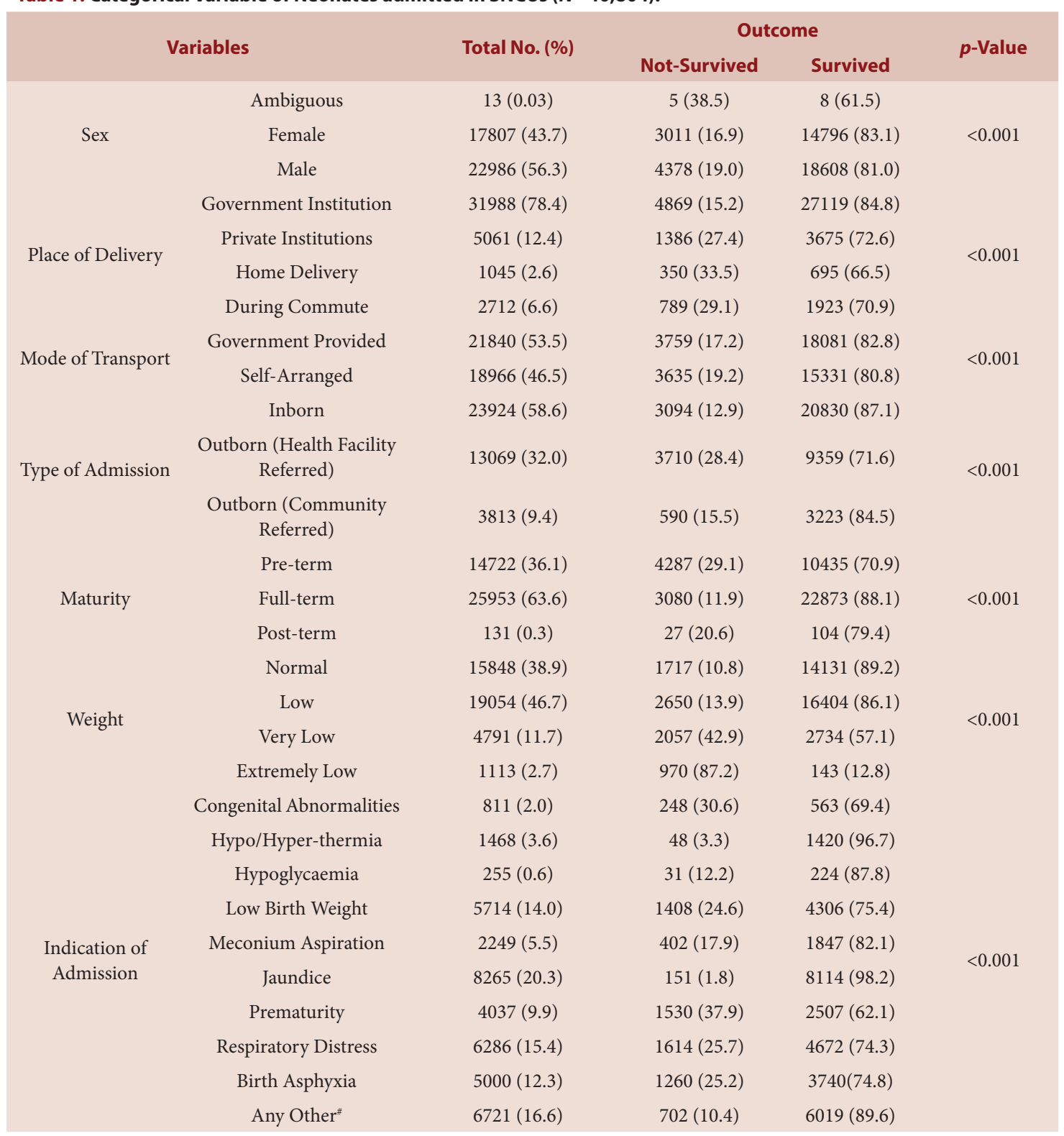

"Hemorrhagic disease of newborn, neonatal tetanus, suspected inborn error of metabolism, Tetanus, Unknown 
Table 2: Regression Equation Model ( 6 categories and 27 sub categories).

\begin{tabular}{|c|c|c|c|c|c|c|c|c|c|}
\hline \multirow{3}{*}{ S.No. } & \multicolumn{9}{|c|}{ Variables in the Equation } \\
\hline & & \multirow{2}{*}{ B } & \multirow{2}{*}{ S.E. } & \multirow{2}{*}{ Wald } & \multirow{2}{*}{ df } & \multirow{2}{*}{ Sig. } & \multirow{2}{*}{ AOR } & \multicolumn{2}{|c|}{ 95\% C.I. for AOR } \\
\hline & & & & & & & & Lower & Upper \\
\hline \multirow[t]{5}{*}{1.} & Category - PoD (FRUs) & & & 53.156 & 4 & .000 & & & \\
\hline & Category - PoD (PHCs) & .532 & .128 & 17.171 & 1 & .000 & 1.702 & 1.324 & 2.189 \\
\hline & Category - PoD (Home Delivery) & -.402 & .081 & 24.403 & 1 & .000 & .669 & .571 & .785 \\
\hline & Category - PoD (Pvt. Institutions) & .083 & .047 & 3.152 & 1 & .076 & 1.087 & .991 & 1.192 \\
\hline & Category - PoD (Others) & .006 & .052 & .012 & 1 & .913 & 1.006 & .908 & 1.114 \\
\hline 2. & Category MoT (Self arranged) & -.128 & .029 & 19.347 & 1 & .000 & .879 & .830 & .931 \\
\hline \multirow[t]{3}{*}{3.} & Category ToA (Inborn) & & & 331.054 & 2 & .000 & & & \\
\hline & $\begin{array}{c}\text { Category ToA (Outborn-Health Facility } \\
\text { Referred) }\end{array}$ & -.587 & .036 & 268.002 & 1 & .000 & .556 & .518 & .596 \\
\hline & $\begin{array}{c}\text { Category ToA (Outborn-Community } \\
\text { Facility Referred) }\end{array}$ & -.028 & .064 & .197 & 1 & .657 & .972 & .857 & 1.102 \\
\hline \multirow[t]{3}{*}{4.} & Category Maturity (Full term) & & & 19.278 & 2 & .000 & & & \\
\hline & Category Maturity (Pre term) & -.155 & .039 & 16.084 & 1 & .000 & .856 & .794 & .924 \\
\hline & Category Maturity (Post term) & -.433 & .221 & 3.823 & 1 & .051 & .649 & .421 & 1.001 \\
\hline \multirow[t]{4}{*}{5.} & Category of Weight (Normal) & & & 1945.136 & 3 & .000 & & & \\
\hline & Category of Weight (Low) & -.191 & .038 & 25.251 & 1 & .000 & .826 & .767 & .890 \\
\hline & Category of Weight (Very Low) & -1.343 & .052 & 656.405 & 1 & .000 & .261 & .235 & .289 \\
\hline & Category of Weight (Extremely Low) & -2.854 & .075 & 1442.699 & 1 & .000 & .058 & .050 & .067 \\
\hline \multirow[t]{11}{*}{6.} & Category of IoA (Any Other) & & & 1377.986 & 9 & .000 & & & \\
\hline & $\begin{array}{c}\text { Category of IoA (Congenital } \\
\text { Abnormalities) }\end{array}$ & -.779 & .082 & 89.611 & 1 & .000 & .459 & .391 & .539 \\
\hline & Category of IoA (Hypo/Hyper-thermia) & .932 & .153 & 37.232 & 1 & .000 & 2.541 & 1.883 & 3.428 \\
\hline & Category of IoA (Hypoglycemia) & -.080 & .199 & .164 & 1 & .686 & .923 & .625 & 1.362 \\
\hline & Category of IoA (Low Birth Weight) & -.039 & .057 & .466 & 1 & .495 & .962 & .859 & 1.076 \\
\hline & Category of IoA (Meconium Aspiration) & -.793 & .068 & 134.050 & 1 & .000 & .452 & .396 & .517 \\
\hline & Category of IoA (Jaundice) & 1.521 & .092 & 273.899 & 1 & .000 & 4.578 & 3.823 & 5.482 \\
\hline & Category of IoA (Prematurity) & -.466 & .061 & 58.050 & 1 & .000 & .628 & .557 & .708 \\
\hline & Category of IoA (Respiratory Distress) & -.684 & .051 & 177.194 & 1 & .000 & .505 & .456 & .558 \\
\hline & Category of IoA (Birth Asphyxia) & -1.068 & .052 & 424.102 & 1 & .000 & .344 & .310 & .380 \\
\hline & Constant & 2.869 & .050 & 3293.886 & 1 & .000 & 17.619 & & \\
\hline
\end{tabular}

motherhood, poor nutrition, sanitation, safe drinking water, low use of antenatal care and skilled intrapartum care aggravates the chances of deaths. ${ }^{11-16}$ The child survival efforts also depend upon the program implementation and their level of coverage across the Districts.

The Gujarat State has achieved considerable reduction in neonatal mortality after the introduction of National Health Mission (NHM). The NHM has supported to create infrastructure, provision of logistics and operational cost to execute neonatal care. There are 43 Govt Supported SNCUs across the Gujarat but the survival rate depends upon wide variety of factors surrounds mothers' antenatal to postnatal care and also on facility to community level new-born care. The analysis of demographic profile and type of admission were similar to previous studies of India. ${ }^{17-20}$ As in this study, six variables- sub variables were analysed mostly on operational programmatic management and their association to neonatal survival suggested that comprehensive package for neonatal care should be in place and monitoring of these variables would support the positive outcome of overall neonatal survival strategies.

The State has to utilize existing strategies of program implementation framework to accelerate the pace of reduction in neonatal mortality. ${ }^{21}$ The strong program monitoring structure has to be established by linking community-based interventions like home based new born care by ASHA to facility based new-born care (SNCU). The concentrated efforts in these identified areas are required starts from demand generation to facility-based care, a continuum care approach for neonates to under 5 in to have uniform achievement of SDG targets across the State.

\section{CONCLUSION}

The survival rate among the neonates depends upon several factors which play significant role in neonatal survival and hence must be given attention along with strengthening of program interventions. The study supports policy makers and program managers to identify the enablers 
and barriers in operational management along with highlighting key preventive areas for efforts.

\section{ACKNOWLEDGEMENT}

SNCUs across Gujarat for the valuable data.

\section{CONFLICTING INTEREST}

The authors declare that there is no conflict of interest.

\section{ABBREVIATIONS}

SNCU: Special newborn care units; U5MR: Under 5 mortality rate; NMR: Neonatal mortality rate; LAMA: Left against medical advice; NHM: National Health mission.

\section{REFERENCES}

1. Sustainable development goals. 2020. https://sustainabledevelopment.un.org/

2. Levels and trends in child mortality: Report. 2018. Estimates Developed by the UN Inter-agency Group for Child Mortality Estimation. New York: UNICEF. 2018.

3. IIPS, ICF. National Family Health Survey (NFHS-4), 2015-16: India. Mumbai: International Institute for Population Sciences. 2017.

4. IIPS, ORC-Macro. National Family Health Survey (NFHS-3), 2005-2006: India. Mumbai: International Institute for Population Sciences. 2007.

5. Chapter no. 4, Sample Registration System 2016, Registrar General of India New Delhi. 2016. Available from http://www.censusindia.gov.in/vital_statistics/ SRS_Reports_2013.html, accessed on 07.05.2019)

6. Lawn JE, Blencowe H, Oza S, You D, Lee AC, Waiswa P, et al. Lancet Every Newborn Study Group. Every Newborn: Progress, priorities and potential beyond survival. Lancet. 2014;384(9938):189-205.

7. Bhutta ZA, Das JK, Bahl R, Lawn JE, Salam RA, Paul VK, et al. Lancet Newborn Interventions Review Group; Lancet Every Newborn Study Group. Can available interventions end preventable deaths in mothers, newborn babies and stillbirths and at what cost?. Lancet. 2014;384(9940):347-70.

8. Dickson KE, Simen-Kapeu A, Kinney MV, Huicho L, Vesel L, Lackritz E, et al. Lancet Every Newborn Study Group. Every Newborn: health-systems bottlenecks and strategies to accelerate scale-up in countries. Lancet. 2014;384(9941):438-54

9. Lopez A. Reducing child mortality. Bulletin of the World Health Organization. 2000;78:1173

10. Bora JK, Saikia N. Neonatal and under-five mortality rate in Indian districts with reference to Sustainable Development Goal 3: An analysis of the National Family Health Survey of India (NFHS), 2015-2016. PLoS One. 2018;13(7):e0201125. https://doi.org/10.1371/journal. pone.0201125

11. Mosley WH, Chen LC. An analytical framework for the study of child survival in developing countries. Population and Development Review. 1984;10(Supplement):25-45.

12. Rosenfield A, Min CJ, Freedman LP. Making motherhood safe in developing countries. New England Journal of Medicine. 2007;356(14):1395-7.

13. Raj A, Saggurti N, Winter M, Labonte A, Decker MR, et al. The effect of maternal child marriage on morbidity and mortality of children under 5 in India: Cross sectional study of a nationally representative sample. British Medical Journal. 2010;340:b4258.

14. Babatunde O. Population and Development. Science. 2011;333:499.

15. Ohlan R. Pattern of regional disparities in socio-economic development in India: District level analysis. Social Indicators Research. 2013;114(3):841-73. 20.

16. Saikia N, Bora JK, Jasilionis D, Shkolnikov VM. Disability divides in India: Evidence from the 2011 census. PloS One. 2016;11(8):e0159809. https://doi. org/10.1371/journal.pone.0159809 PMID: 27490469

17. Bhatia BD, Mathur NB, Chaturvedi P, Dubey AP. Neonatal mortality pattern in rural based Medical college hospital. Indian J Pediatr.1984;51(3):309-12.

18. Investigators of the National Neonatal Perinatal Database (NNPD), National Neonatology Forum of India (see Appendix for complete list of Investigators). Morbidity and mortality among out born neonates at 10 tertiary care institutions in India during the year 2000. J Trop Pediatr. 2004;50(3):170-4.

19. Rakholia R, Rawat V, Bano M, Singh G. Neonatal morbidity and mortality of sick newborns admitted in a teaching hospital of Uttarakhand. CHRISMED J Health Res. 2014;1(4):228-34.

20. Sridhar PV, Thammanna PS, Sandeep M. Morbidity Pattern and Hospital Outcome of Neonates Admitted in a Tertiary Care Teaching Hospital, Mandya. Int J Sci Stud. 2015;3(6):126-9.

21. India Newborn Action Plan, Child Health Division, Ministry of Health and Family Welfare, Govt of India. 2014. (available from nrhm.gov.in/india-newborn-actionplan.html, access on 07.05.2019). 